\title{
A Fiber Scattering Model with Non-Separable Lobes
}

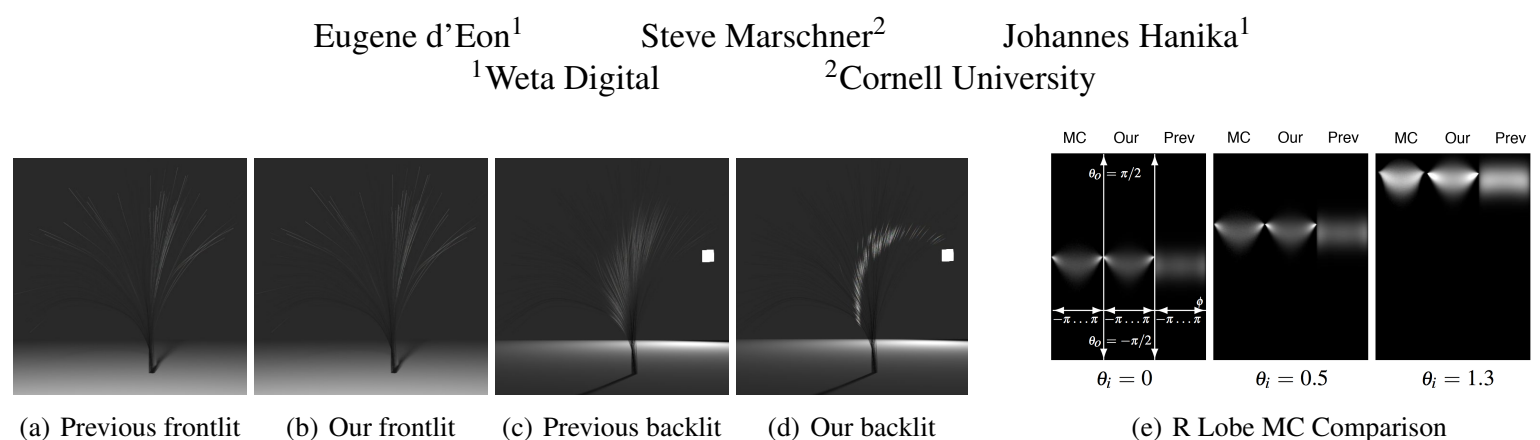

(a) Previous frontlit

(b) Our frontlit

(c) Previous backlit

(d) Our backlit

(e) R Lobe MC Comparison

Figure 1: Glossy reflections from fibers exhibit a light- and view-dependent lobe tightening similar to surface reflectance that previous analytic fiber models lack $(a, c)$. We derive new non-separable fiber-scattering lobes that exhibit this and additional behaviours seen in ground-truth simulation of rough dielectric fiber scattering. Parameter tweaking of previous models can produce results similar to (d), but no previous model with fixed settings can simultaneously produce both $(b)$ and $(d)$. We found that ground-truth renders using explicit cylindrical geometry with Cook Torrance reflectance to be indistinguishable from $(b)$ and $(d)$ (upon convergence, which required significantly more samples per pixel). The difference between the old and new models is further illustrated in (e) by visualizing the R lobe of a circular fiber with Beckmann micro roughness and tilted scales (black hair). In the backlit case (c,d) light grazes the edges of fibers and exhibits the brighter more focused response (seen in (e) where the lobe compresses and brightens for high $|\phi|$ ).

Introduction An important feature of physically-based surface reflectance models is the focusing and increased brightness of glossy lobes at grazing angles. This affect has not been accounted for by previous analytic bidirectional curve scattering distribution functions (BCSDFs) that describe the far-field radiometry of fiber reflectance. We present an approach for incorporating these and other missing behaviours into a practical analytic fiber reflectance model using non-separable lobe functions. We find the visual impact of these effects significant (Figure 1). Our new model is successfully validated against a novel Monte Carlo ground truth analysis for scattering within rough dielectric cylinders.

Our approach Like prior work we describe fiber reflectance $S$ as a sum of lobes $S_{p}$, each of which is factored into a longitudinal scattering function $M_{p}$ and an azimuthal scattering function $N_{p}, S_{p}\left(\theta_{i}, \theta_{o}, \phi\right)=M_{p}\left(\theta_{i}, \theta_{o}, \phi\right) N_{p}\left(\theta_{i}, \theta_{o}, \phi\right)$, following Marschner's [2003] original notation. In previous work, $M_{p}$ has been assumed independent of $\phi$, and $N_{p}$ depends only weakly on the $\theta \mathrm{s}$. In this sense the lobes $S_{p}$ are partly separable. Our approach generalizes the derivation of the Marschner model using a novel Nusselt sphere analysis to accurately estimate the longitudinal mean and variance of each lobe given any relative azimuth $\phi$ between source and detector. These quantities appear in the new model's $M$ functions as new specular cone angles $\theta_{\text {cone }_{p}}(\phi)$, and variances $v_{p}(\phi)$, thus producing a fundamentally azimuthallydependent (and thus, non-separable) model.

New model summary Of all the lobes, the primary reflectance $(\mathrm{R})$ lobe, important for rough conductive fibers as well as dielectric fibers, such as hair, sees the most significant appearance change in our new model. The surface lobe focusing effect (Figure 1) exhibits as a cosine modulation of the fiber $\mathrm{M}$ width $\sqrt{v_{R}}=\beta \sqrt{2} \cos (\phi / 2)$ as opposed to the arbitrary parameter $\sqrt{v_{R}}=\beta_{R}$ of previous models. In the case of tilted scales for hair or fur, we also find a new, more accurate specular cone behaviour for the $\mathrm{R}$ lobe (given scale tilt $\alpha$ )

$$
\begin{aligned}
& \theta_{\text {cone }_{R}}= \\
& -\arcsin \left(\sin \theta_{i}-2 \sin \alpha\left(\cos (\phi / 2) \cos \alpha \cos \theta_{i}+\sin \alpha \sin \theta_{i}\right)\right)
\end{aligned}
$$

which varies the offset of the R and TRT highlights with $\phi$. In the supplementary material we also derive new TT and TRT lobes and discuss an iterative approximate importance sampling scheme for sampling non-separable products.

In practice Our new model is practical and efficient and has been applied in film production for rendering hair. We have found the main strength of non-separable fiber scattering to be the consistent appearance of fibers for moving or multiple light sources-many previous fiber models, when tweaked, can create very believable results for any single static light, but the view- and light-dependent focusing of glossy reflectance from surfaces requires an analog behaviour for far field fiber reflectance, thus requiring non-separable lobes for maintaining consistent appearance and for seamless LOD transitions from near and far-field fiber representations. We further expect our approach to find use in future investigations of elliptical hair reflectance functions, where non-separable behaviours have been recently noted in measured hair reflectance data [Khungurn and Marschner 2014], as well as for cloth reflectance, where glossy threads will likewise yield non-separable behaviours.

\section{References}

KHUngurn, P., AND MARschner, S. 2014. On azimuthal scattering behavior in hair fibers. Accepted to TOG.

Marschner, S. R., Jensen, H. W., Cammarano, M., WorLEY, S., AND HANRAHAN, P. 2003. Light scattering from human hair fibers. In SIGGRAPH '03: ACM SIGGRAPH 2003 Papers, ACM, New York, NY, USA, 780-791.
Permission to make digital or hard copies of part or all of this work for personal or classroom use is granted without fee provided that copies are not made or distributed for commercial advantage and that copies bear this notice and the full citation on the first page. Copyrights for third-party components of this work must be honored. For all other uses, contact the Owner/Author.

SIGGRAPH 2014, August $10-14,2014$, Vancouver, British Columbia, Canada.

2014 Copyright held by the Owner/Author.

ACM 978-1-4503-2960-6/14/08 